Nuclear Physics A444 (1985) 189-208

(C) North-Holland Publishing Company

\title{
COHERENT-STATE THEORY FOR THE LST QUASISPIN GROUP
}

\author{
K.T. HECHT* \\ Physics Department, University of Michigan, Ann Arbor, Michigan 48109, USA
}

Received 1 May 1985

\begin{abstract}
An extended coherent-state theory is used to give a simple construction for the matrix elements of the $L=0$ pair operators of the $L S T S O(8) \supset \mathrm{U}(4)$ quasispin algebra leading to a more explicit construction of $n$-nucleon states in the orbital seniority scheme.
\end{abstract}

\section{Introduction}

Recent developments ${ }^{1-4}$ ) in the theory of the noncompact symplectic groups $\operatorname{Sp}(2 d, \mathrm{R})$, and more specifically $\operatorname{Sp}(6, \mathrm{R})$ through its application to the nuclear collective model ${ }^{5,6}$ ), have opened up new possibilities for the study of collective motion in terms of the underlying nucleon degrees of freedom ${ }^{7}$ ). In particular, very useful explicit constructions have been given for the discrete infinite-dimensional unitary irreducible representations of $\mathrm{Sp}(2 d, \mathrm{R})$. The cases $\mathrm{Sp}(4, \mathrm{R}) \supset[\mathrm{U}(1) \times \mathrm{SU}(2)]$ and $\operatorname{Sp}(6, R) \supset U(3)$ have been worked out in full detail. The perspective and language of different workers is slightly different. Moshinsky and coworkers ${ }^{2}$ ) emphasize boson realizations. Rowe and Rosensteel ${ }^{1}$ ) have developed an extended coherent-state theory with its associated functional representations in a multidimensional Bargmann space. Deenen and Quesne ${ }^{3}$ ) have introduced the language of partially coherent states to generalize the simple coherent-state theory applicable to $\mathrm{Sp}(2, \mathrm{R})$. Many of the compact groups of interest in nuclear spectroscopy have also been discussed in terms of coherent-state thcory by Dobaczewski ${ }^{8}$ ) in his functional representation analysis of boson expansion theories. To date, however, detailed constructions and applications have been limited to the symplectic groups. The generalized coherent-state methods of refs. ${ }^{1-3}$ ) can be applied with equal ease to many other groups of interest in nuclear spectroscopy, particularly the so-called noninvariance groups ${ }^{9}$ ), which include fermion pair-creation and pair-annihilation operators among their generators. The proton-neutron quasispin group is a unitary symplectic group in four dimensions, the compact analog of $\operatorname{Sp}(4, R)$, and has recently been reexamined in terms of the new generalized coherent-state

*Supported by the US National Science Foundation. 
techniques $\left.{ }^{10}\right)$. The results for $\operatorname{Sp}(6, R)$ have also been extended to the $U S p(6)$ symmetry group ${ }^{11}$ ), in particular in connection with the Ginocchio model ${ }^{12}$ ), and attempts to find a sound fermionic foundation for the rotational or SU(3) limit of the interacting boson model. The generalized coherent-state methods developed for the explicit construction of an orthonormal basis for the noncompact group $\mathrm{Sp}(2 d, \mathrm{R}) \supset \mathrm{U}(d)$ can be taken over directly for any compact noninvariance group with a parallel $\mathrm{G} \supset \mathrm{H}$ group structure. However, these methods are most useful in those cases where the Wigner-Racah calculus for the subgroup $\mathrm{H}$ is worked out in sufficient detail. Another example in this category is the $L S T$ pairing symmetry, $\mathrm{SO}(8) \supset \mathrm{U}(4)$, in its application to the Wigner-supermultiplet nucleon-number classification in the orbital seniority scheme. Since this symmetry has no exact $\operatorname{Sp}(2 d, \mathrm{R})$ $\supset \mathrm{U}(d)$ analog, it is of particular interest to show how the coherent-state methods can be applied to this case.

The quasispin group in the $L S T$ scheme has been identified as an $\mathrm{SO}(8) \supset \mathrm{U}(4)$ symmetry group by Flowers and Szpikowski ${ }^{13}$ ) and applied to $L S T$ pairing theory by Pang ${ }^{14}$ ). However, the applications have been very limited since the details of the theoretical apparatus could be worked out only for the lowest-seniority states $v=0$ and $v=1$. It is the purpose of the present contribution to show how the new coherent-state techniques make possible a generalization to states of arbitrary seniority and reduced Wigner-supermultiplet symmetry.

The coherent-state realization of the $L S T$ quasispin algebra will be given in sect. 2. As in refs. ${ }^{1-3}$ ) coherent-state theory is used to express the generators of the U(4) subalgebra of the full $\mathrm{SO}(8)$ Lie algebra in terms of a set of U(4) "intrinsic" generators and a set of U(4) "collective" generators. The intrinsic generators act only in the subspace of $v$ nucleons coupled to reduced Wigner-supermultiplet symmetry $\left(p p^{\prime} p^{\prime \prime}\right)$. Following ref. $\left.{ }^{1}\right)$ the collective generators will be expressed in terms of a set of six Bargmann-space variables $z_{i}(i=1, \ldots 6)$ and their adjoint derivative operators $\partial / \partial z_{i}$. The strategy used to calculate the matrix elements of the full $\operatorname{SO}(8)$ quasispin algebra is again that of refs. ${ }^{1-3}$ ). One considers first a simpler algebra, a direct sum of a six-dimensional Heisenberg-Weyl algebra and the $U(4)$ algebra of intrinsic generators. The Heisenberg-Weyl algebra is generated by the six collective operators $z_{i}$ and the six $\nabla_{i}$, a realization of a set of six boson creation and annihilation operators. The very explicit construction of this algebra in terms of Bargmann-space solid harmonics is given in sect. 2 . As in refs. ${ }^{1-3,10}$ ) it leads to a very simple analytical expression for the matrix elements of the $L=0$ pair-creation/annihilation operators valid for all cases where both ket and bra states are multiplicity-free with respect to the $\mathrm{U}(4)$ algebra; i.e. where, for given seniority number $v$ and reduced Wigner supermultiplet quantum numbers $\left(p p^{\prime} p^{\prime \prime}\right)$, the states are completely specified by nucleon number $n$ and the Wigner supermultiplet quantum numbers of the full $n$-nucleon system. As in the earlier applications, the more general case involves the square-root-taking of a hermitean $\mathrm{U}(4)$-invariant operator (or matrix) $\kappa^{2}$ which converts states specified by both $\mathrm{U}(4)$ intrinsic and $\mathrm{U}(4)$ collective labels from a mere 
labelling scheme into an orthonormal unitary basis for the $S O(8) \supset U(4)$ symmetry group. This case is discussed with a detailed example in sect. 3. In the most general case some additional work was required to construct the needed SU(4) Wigner and Racah coefficients. The coherent-state methods and the Bargmann-space constructions have again proved very useful. Sect. 4 shows how these methods can be used in the general case where the spin and isospin quantum numbers $S$ and $T$ are not sufficient to label the states of the Wigner supermultiplet.

\section{Coherent-state realization of the $L S T$ quasispin group}

In the $L S T$ scheme the quasispin group includes the six pair-creation (and pair-annihilation) operators coupled to $L=0, S T=10$ and 01 ,

$$
\begin{aligned}
2 S+1,2 T+1 & A^{\dagger}\left(M_{S}, M_{T}\right)=\left[a^{\dagger} \times a^{\dagger}\right]_{0}^{L=0, S, T} M_{S}, M_{T}, \\
{ }^{2 S+1,2 T+1} A\left(M_{S}, M_{T}\right) & =\left({ }^{2 S+1,2 T+1} A^{\dagger}\left(M_{S}, M_{T}\right)\right)^{\dagger},
\end{aligned}
$$

[The notations and normalizations follow ref. ${ }^{14}$ ).] Together with the Wigner-supermultiplet generators

$$
\boldsymbol{S}, \quad \boldsymbol{T}, \quad(\sigma \tau)_{m_{s} m_{t}}=\sum_{r=1}^{n} \sigma(r)_{m_{s}} \tau(r)_{m_{t}},
$$

and the nucleon-number operator, which appears naturally in the combination $\frac{1}{2} N_{\mathrm{op}}-\Omega$, these generate the $L S T$ quasispin group $\mathrm{SO}(8) \supset \mathrm{U}(4)$ [see table 1 of ref. $\left.{ }^{14}\right)$ ]. In eq. (2), $r$ is the particle index, and $\Omega=\Sigma(2 l+1)$ may involve a single term or a sum over active $l$-subshells. As in refs. ${ }^{1-3,10}$ ), it will be useful to convert these generators into cartesian components in the separate spin and isospin spaces; e.g. ${ }^{31} A^{\dagger}( \pm 1,0)=\mp \sqrt{\frac{1}{2}}\left({ }^{31} A_{1,0}^{\dagger} \pm i^{31} A_{2,0}^{\dagger}\right)$. In terms of such cartesian components, the commutator algebra of $\mathrm{SO}(8)$ is given by

$$
\begin{aligned}
{\left[{ }^{31} A_{i, 0},{ }^{31} A_{j, 0}^{\dagger}\right] } & =\delta_{i j}\left(\Omega-\frac{1}{2} N_{\mathrm{op}}\right)+i \varepsilon_{i j k} S_{k}, \\
{\left[{ }^{13} A_{0, i},{ }^{13} A_{0, j}^{\dagger}\right] } & =\delta_{i j}\left(\Omega-\frac{1}{2} N_{\mathrm{op}}\right)+i \varepsilon_{i j k} T_{k}, \\
{\left[{ }^{31} \boldsymbol{A}, \boldsymbol{T}\right] } & =\left[{ }^{13} \boldsymbol{A}, \boldsymbol{S}\right]=0, \\
{\left[{ }^{31} A_{\imath, 0}, S_{j}\right] } & =i \varepsilon_{i j k}{ }^{31} A_{k, 0}, \\
{\left[{ }^{13} A_{0, i}, T_{j}\right] } & \left.=i \varepsilon_{i j k}{ }^{13} A_{0, k},\right\} \\
{\left[{ }^{31} A_{i, 0}^{\dagger},{ }^{13} A_{0, j}\right] } & =\left[{ }^{13} A_{0, j}^{\dagger},{ }^{31} A_{t, 0}\right]=\frac{1}{2}(\sigma \tau)_{i, j}, \\
{\left[\frac{1}{2}(\boldsymbol{\sigma} \tau)_{i, j},{ }^{31} A_{k, 0}\right] } & =-\delta_{i k}{ }^{13} A_{0, j}, \\
{\left[\frac{1}{2}(\sigma \tau)_{i, j},{ }^{13} A_{0, k}\right] } & =-\delta_{j k}{ }^{31} A_{i, 0},
\end{aligned}
$$

with $i, j, k=1,2,3$. 
The $n$-particle state vectors in the $L S T$ seniority scheme can be constructed from linear combinations of states:

$$
|\psi\rangle=\mathscr{N}\left[\left[A^{\dagger} \times A^{\dagger} \times \cdots \times A^{\dagger}\right]^{\left[f_{P}\right]}\left|n=v\left[f_{v}\right]\right\rangle\right]_{S M_{S} T M_{T}}^{[f]},
$$

where $\mathscr{N}$ is a normalization factor. The $v$ nucleons, free of pairs coupled to $L=0$, i.e. with

$$
\boldsymbol{A}\left|n=v\left[f_{v}\right]\right\rangle=0
$$

have $\mathrm{U}(4)$ symmetry given by a Young tableau $\left[f_{v}\right]$, with equivalent $\mathrm{SO}(6)$ quantum numbers $\left(p p^{\prime} p^{\prime \prime}\right)$,

$$
\begin{aligned}
p & =\frac{1}{2}\left(f_{v_{1}}+f_{v_{2}}-f_{v_{3}}-f_{v_{4}}\right), \\
p^{\prime} & =\frac{1}{2}\left(f_{v_{1}}-f_{v_{2}}+f_{v_{3}}-f_{v_{4}}\right), \\
p^{\prime \prime} & =\frac{1}{2}\left(f_{v_{1}}-f_{v_{2}}-f_{v_{3}}+f_{v_{4}}\right), \quad v=\sum_{i=1}^{4} f_{v_{i}},
\end{aligned}
$$

These $v$-particle states are acted upon by $\frac{1}{2}(n-v)(L=0)$-coupled pair-creation operators $\boldsymbol{A}^{\dagger}$ which are coupled to $\mathrm{U}(4)$ symmetry described by a Young tableau $\left[f_{P}\right]$ with $a=\frac{1}{4}(n-v-2 P)$ columns of 4 and $P$ columns of 2 , with equivalent $\mathrm{SO}(6)$ symmetry $(P 00)$. The $\left[f_{v}\right] \times\left[f_{P}\right] \mathrm{U}(4)$ coupling leads to the resultant $n$-particle $U(4)$ symmetry $[f]$. Note that the $\left[f_{P}\right]$ only form a convenient labeling, since the labels $a$ and $P$ cannot be associated directly with the eigenvalues of a hermitean operator.

Generalized coherent states for the space of the above $|\psi\rangle$ will be constructed following both the methods and the language of ref. ${ }^{1}$ ). For this purpose it is useful to introduce six complex (Bargmann-space) variables $z_{i}$, to be associated with the six components of the pair-annihilation operators. These can be organized into a 6-vector to be denoted by $z$, or two 3 -vectors, $z_{\sigma}, z_{\tau}$, one in spin space and one in isospin space:

$$
\left(\begin{array}{c}
{ }^{31} A \\
{ }^{13} A
\end{array}\right) \rightarrow\left(\begin{array}{l}
z_{\sigma} \\
z_{\tau}
\end{array}\right) \equiv z \equiv\left(\begin{array}{l}
z_{1} \\
z_{2} \\
z_{3} \\
z_{4} \\
z_{5} \\
z_{6}
\end{array}\right) .
$$

In terms of these the generalized coherent state can be expressed as

$$
\left|z, v\left(p p^{\prime} p^{\prime \prime}\right)\right\rangle=\exp \left(z^{*} \cdot A^{\dagger}\right)\left|v\left(p p^{\prime} p^{\prime \prime}\right)\right\rangle \text {. }
$$

In a shorthand notation, eq. (8) represents a family of coherent states, one for each subgroup label of $\left(p p^{\prime} p^{\prime \prime}\right)$; but these subgroup labels have been suppressed. Since all final-state vectors will be SU(4)-coupled, and since all matrix elements will be expressed in terms of SU(4) reduced matrix elcments, this distinction between a simple coherent state and a family of generalized coherent states [or partially coherent states in the language of ref. $^{2}$ )] will be suffused in the notation. 
The state vectors $|\psi\rangle$ have the functional representation ${ }^{1}$ )

$$
\psi_{v\left(p p^{\prime} p^{\prime \prime}\right)}(z)=\left\langle v\left(p p^{\prime} p^{\prime \prime}\right)|\exp (z \cdot A)| \psi\right\rangle,
$$

and operators $\mathcal{O}$ are represented by $\Gamma(\mathcal{O})$ :

$$
\begin{aligned}
\Gamma(\mathcal{O}) \psi_{v\left(p p^{\prime} p^{\prime \prime}\right)}(z)= & \left\langle v\left(p p^{\prime} p^{\prime \prime}\right)|\exp (z \cdot A) \mathcal{O}| \psi\right\rangle \\
= & \left\langle v\left(p p^{\prime} p^{\prime \prime}\right)\right|\left(\mathcal{O}+[(z \cdot A), \mathcal{O}]+\frac{1}{2}[(z \cdot A),[(z \cdot A), \mathcal{O}]]+\cdots\right) \\
& \times \exp (z \cdot A)|\psi\rangle .
\end{aligned}
$$

This leads to the coherent-state realization of the $\mathrm{SO}(8)$ algebra, a (nonunitary) Dyson realization:

$$
\begin{aligned}
\Gamma\left({ }^{31} A\right)= & \frac{\partial}{\partial z_{\sigma}} \equiv \nabla_{\sigma}, \quad \Gamma\left({ }^{13} A\right)=\frac{\partial}{\partial z_{\tau}} \equiv \nabla_{\tau}, \\
\Gamma(S)= & \sigma-i\left[z_{\sigma} \times \nabla_{\sigma}\right], \quad \Gamma(T)=t-i\left[z_{\tau} \times \nabla_{\tau}\right], \\
\Gamma\left(\frac{1}{2}(\sigma \tau){ }_{i j}\right)= & \frac{1}{2}(\sigma \tau)_{i j}+z_{\sigma_{\tau}} \nabla_{\tau}+z_{\tau_{j}} \nabla_{\sigma_{\tau}}, \\
\Gamma\left(\frac{1}{2} N_{\mathrm{op}}-\Omega\right)= & \left(\frac{1}{2} v-\Omega\right) \mathbf{1}+z_{\sigma} \cdot \nabla_{\tau}+z_{\tau} \cdot \nabla_{\sigma}, \\
\Gamma\left({ }^{31} A^{\dagger}\right)= & \left(\Omega-\frac{1}{2} v\right) z_{\sigma}-i\left[z_{\sigma} \times \sigma\right]-\frac{1}{2} \sigma\left(z_{\tau} \cdot \tau\right) \\
& +\frac{1}{2}\left(z_{\sigma} \cdot z_{\sigma}-z_{\tau} \cdot z_{\tau}\right) \nabla_{\sigma}-z_{\sigma}\left(z_{\sigma} \cdot \nabla_{\sigma}+z_{\tau} \cdot \nabla_{\tau}\right), \\
\Gamma\left({ }^{13} A^{\dagger}\right)= & \left(\Omega-\frac{1}{2} v\right) z_{\tau}-i\left[z_{\tau} \times t\right]-\frac{1}{2}\left(z_{\sigma} \cdot \sigma\right) \tau \\
& +\frac{1}{2}\left(z_{\tau} \cdot z_{\tau}-z_{\sigma} \cdot z_{\sigma}\right) \nabla_{\tau}-z_{\tau}\left(z_{\sigma} \cdot \nabla_{\sigma}+z_{\tau} \cdot \nabla_{\tau}\right),
\end{aligned}
$$

where $\sigma\left(z_{\tau} \cdot \tau\right)$, for example, is shorthand for $\left(z_{\tau}\right)_{\alpha}(\sigma \tau)_{i \alpha}$ with summation convention for repeated indices. Note also that the U(4) subalgebra, generated by

$$
S, \quad T, \quad \frac{1}{2}(\sigma \tau)_{i j}, \quad\left(\frac{1}{2} N_{\text {op }}-\Omega\right),
$$

is a direct sum of an "intrinsic" $U(4)$ algebra

$$
\circ, \quad t, \quad \frac{1}{2}(\sigma \tau)_{i j}, \quad\left(\frac{1}{2} v-\Omega\right) \mathbb{t},
$$

acting on the subspace $\left|v\left(p p^{\prime} p^{\prime \prime}\right) \ldots\right\rangle$, and a set of "collective" $\mathrm{U}(4)$ generators expressed in terms of the six Bargmann-space variables $z$ and their derivative operators $\nabla$.

The SU(4) Casimir operator can thus be written as

$$
\begin{aligned}
C_{\text {full }}= & S^{2}+T^{2}+\frac{1}{4}(\sigma \tau)_{\alpha \beta}(\sigma \tau)_{\alpha \beta} \\
= & C_{\text {intrinsic }}+C_{\text {collective }}-2 i\left\{\left(\sigma \cdot\left[z_{\sigma} \times \nabla_{\sigma}\right]\right)+\left(t \cdot\left[z_{\tau} \times \nabla_{\tau}\right]\right)\right\} \\
& +(\sigma \tau)_{\alpha \beta}\left(z_{\sigma_{\alpha}} \nabla_{\tau_{\beta}}+z_{\tau_{\beta}} \nabla_{\sigma_{\alpha}}\right),
\end{aligned}
$$


with

$$
\begin{aligned}
& C_{\text {intrinsic }}=\sigma^{2}+\ell+\frac{1}{4}(\sigma \tau)_{\alpha \beta}(\sigma \tau)_{\alpha \beta}, \\
& C_{\text {collective }}=\left(z_{\tau}^{2}-z_{\sigma}^{2}\right)\left(\nabla_{\sigma}^{2}-\nabla_{\tau}^{2}\right)+\left(z_{\sigma} \cdot \nabla_{\sigma}+z_{\tau} \cdot \nabla_{\tau}\right)\left(z_{\sigma} \cdot \nabla_{\sigma}+z_{\tau} \cdot \nabla_{\tau}+4\right),
\end{aligned}
$$

where, for example, $z_{\tau}^{2} \equiv\left(z_{\tau} \cdot z_{\tau}\right)$. In the method of refs. $\left.{ }^{1-3}\right)$ one considers first the simpler algebra

$$
\begin{gathered}
z_{\sigma}, z_{\tau}, \quad \nabla_{\sigma}, \nabla_{\tau}, \\
\sigma, \quad t, \quad \frac{1}{2}(\sigma \tau)_{i j}, \quad\left(\frac{1}{2} v-\Omega\right) \boldsymbol{0} .
\end{gathered}
$$

Since the six $z$ and $\nabla$ operators commute with the intrinsic operators, this is a direct sum of a 6-dimensional Heisenberg-Weyl algebra and the U(4) intrinsic algebra. In Bargmann space the six operators $z=\left(z_{\sigma}, z_{\tau}\right)$ and the six $\nabla=\left(\nabla_{\sigma}, \nabla_{\tau}\right)$ are a realization of a set of six boson creation and annihilation operators. Due to the Bargmann measure in the complex $z$-spaces the operators $\nabla_{i}$ are the hermitean adjoints of $z_{i}$. The first step of the calculation involves the evaluation of the SU(4) (or the equivalent $\mathrm{SO}(6)$ ) reduced matrix elements of these 6-dimensional bosonic operators. These follow from an explicit construction of the Bargmann-space $U(4) \supset$ $\mathrm{SU}(4) \supset\left[\mathrm{SU}(2)_{S} \times \mathrm{SU}(2)_{T}\right]$ polynomials of degree $2 a+P$ in the $z$, with $n-v=4 a$ $+2 P$, i.e. with Young tableaux with $a=\frac{1}{4}(n-v-2 P)$ columns of 4 , and $P$ columns of 2 and hence $S U(4)$ symmetry $[P P]$ (with equivalent $S O(6)$ quantum numbers (P00)). These have the specific form

$$
\begin{aligned}
& \psi_{S M_{S}, T M_{\tau}}^{a(P 00)}\left(z_{\sigma}, z_{\tau}\right)=\left[\frac{(P+2) !}{a ! 2^{2 a}(P+a+2) !}\right]^{1 / 2}\left(z_{s}^{2}-z_{\tau}^{2}\right)^{a} \\
& \times \sum_{k=0}^{\alpha} \frac{1}{2^{\alpha}}\left[\frac{\alpha !(S+k) !(T+\alpha-k) !(2 T+2 \alpha+1) !(2 S+2 \alpha+1) !(S+T+\alpha+1) !}{(\alpha-k) ! k !(2 S+2 k+1) !(2 T+2 \alpha-2 k+1) !(T+\alpha) !(S+\alpha) !(S+T+2 \alpha+1) !}\right]^{1 / 2} \\
& \times P_{S M_{S}}^{(2 k+S .0)}\left(z_{\sigma}\right) P_{T M_{\tau}}^{(2 \alpha-2 k+T, 0)}\left(z_{\tau}\right),
\end{aligned}
$$

with

$$
\alpha=\frac{1}{2}(P-S-T) .
$$

In eq. (14), the $P_{J M}^{(0,0)}(z)$ are the Bargmann transforms of normalized, 3-dimensional harmonic-oscillator functions [see refs. ${ }^{15,16}$ ); the phase convention is that of ref. $\left.{ }^{16}\right)$ ].

$$
P_{J M}^{(Q, 0)}(z)=\left[\frac{4 \pi 2^{J}\left(\frac{1}{2}(Q+J)\right) !}{\left(\frac{1}{2}(Q-J)\right) !(Q+J+1) !}\right]^{1 / 2}(z \cdot z)^{(Q-J) / 2} \mathscr{Y}_{J M}(z) .
$$

where $\mathscr{Y}_{J M}(z)$ is a standard normalized solid harmonic in $z$. To arrive at eq. (14) it is sufficient to consider states with $M_{S}=S, M_{T}=T$. The eigenvalue equation

$$
C_{\text {collective }} \psi_{S S, T T}^{a(P O)}\left(z_{\sigma}, z_{\tau}\right)=P(P+4) \psi_{S S, T T}^{a(P O)}\left(z_{\sigma}, z_{\tau}\right) \text {. }
$$


with

$$
\begin{aligned}
\psi_{S S, T T}^{a(P 00)}\left(z_{\sigma}, z_{\tau}\right)= & \left(z_{\sigma}^{2}-z_{\tau}^{2}\right)^{a} \sum_{k=0}^{\alpha} c_{k}\left(z_{\sigma}^{2}\right)^{k}\left(z_{\tau}^{2}\right)^{\alpha-k} \\
& \times(-1)^{S+T}\left(z_{\sigma_{1}}+i z_{\sigma_{2}}\right)^{S}\left(z_{\tau_{1}}+i z_{\tau_{2}}\right)^{T}
\end{aligned}
$$

leads to a recursion relation for the $c_{k}$. This can be solved to yield

$$
c_{k}=\frac{\alpha !(2 S+1) ! !(2 T+2 \alpha+1) ! !}{(\alpha-k) ! k !(2 S+2 k+1) ! !(2 T+2 \alpha-2 k+1) ! !} c_{0} .
$$

Finally, the evaluation of the normalization factor for eq. (14) is carried out in two steps, first for the $\mathrm{SU}(4) \supset\left[\mathrm{SU}(2)_{S} \times \mathrm{SU}(2)_{T}\right]$ factor with $a=0$, and second for the $S, T$-independent $\mathrm{U}(4) \supset \mathrm{SU}(4)$ factor.

To evaluate the SU(4) reduced matrix elements of $z$ and $\nabla$ it is sufficient to use states with $S M_{S}, T M_{T}=P P, 00 ; P(P-2), 00 ;(P-2)(P-2), 00$; or similar simple combinations. The action of simple operators such as $\left(z_{\sigma 1} \pm i z_{\sigma 2}\right)$ on such simple states yield the SU(4) reduced matrix elements

$$
\begin{aligned}
\langle a P+1\|z\| a P\rangle & =\left[\frac{(P+1)(a+P+3)}{(P+3)}\right]^{1 / 2}, \\
\langle a+1 P-1\|z\| a P\rangle & =-\left[\frac{(a+1)(P+3)}{(P+1)}\right]^{1 / 2}, \\
\langle a P-1\|\nabla\| a P\rangle & =-\left[\frac{(P+3)(a+P+2)}{(P+1)}\right]^{1 / 2}, \\
\langle a-1 P+1\|\nabla\| a P\rangle & =\left[\frac{a(P+1)}{(P+3)}\right]^{1 / 2},
\end{aligned}
$$

with $a=\frac{1}{4}(n-v-2 P)$. Reduced matrix elements are defined by (full matrix element $)=($ double-barred reduced matrix element $) \times(S U(4)$ Wigner coefficient $)$, without square roots of dimensional factors. The SU(4) Wigner coefficients are products of $\mathrm{SU}(4) \supset\left[\mathrm{SU}(2)_{S} \times \mathrm{SU}(2)_{T}\right]$ coefficients [see table A.2.1 of ref. $\left.{ }^{17}\right)$ ] and ordinary $S U(2)$ spin and isospin Wigner coefficients.

Straightforward unitary group Racah algebra gives the $S U(4)$ reduced matrix element of the collective variable $z$ in the SU(4)-coupled space of [intrinsic $\times$ collective] degrees of freedom:

$$
\begin{aligned}
\left\langle n+2, v\left[\left(p p^{\prime} p^{\prime \prime}\right) \times(P \pm 1,00)\right]\left(P_{\mathrm{L}} P_{\mathrm{L}}^{\prime} P_{\mathrm{L}}^{\prime \prime}\right)\|z\| n, v\left[\left(p p^{\prime} p^{\prime \prime}\right) \times(P 00)\right]\left(P_{\mathrm{R}} P_{\mathrm{R}}^{\prime} P_{\mathrm{R}}^{\prime \prime}\right)\right\rangle \\
\quad \equiv\left\langle n+2, v\left[\left[f_{v}\right] \times[P \pm 1, P \pm 1]\right]\left[f_{\mathrm{L}}\right]\|z\| n, v\left[\left[f_{v}\right] \times[P P]\right]\left[f_{\mathrm{R}}\right]\right\rangle \\
\quad=\langle n+2(P \pm 1)\|z\| n P\rangle U\left(\left[f_{v}\right][P P]\left[f_{\mathrm{L}}\right][11] ;\left[f_{\mathrm{R}}\right][P \pm 1, P \pm 1]\right)
\end{aligned}
$$


where the $U$-coefficient is a multiplicity-free $\mathrm{SU}(4)$ Racah coefficient (in unitary form). In eq. (19) the SU(4)-coupled [intrinsic $\times$ collective] state vectors are given both in $\mathrm{SO}(6)$ and $\mathrm{U}(4)$ language. The needed SU(4) Racah coefficients are not known in complete generality. For many cases of actual interest, however, involving simple $S U(4)$ representations $\left[f_{v}\right]$, all SU(4) representations in the $U$-coefficient involve at most 3-rowed tableaux or can be related to such a 3-rowed tableau coefficient by a symmetry relation. Such 3-rowed tableau coefficients are known from SU(3) tabulations. In other cases the needed SU(4) coefficients can be calculated from the tabulations of ref. ${ }^{17}$ ). For most cases of practical interest the matrix elements of eq. (20) are therefore evaluated in terms of easily calculated coefficients.

Since the Dyson realization, $\Gamma$, of the $\mathrm{SO}(8)$ algebra, as given by eqs. (11), is nonunitary, the final step in the process of constructing a unitary basis for the $\mathrm{SO}(8)$ algebra involves a transformation to a unitary or Holstein-Primakoff realization, $\gamma$, of this algebra. As in refs. ${ }^{1-3}$ ) this is achieved by means of a hermitean U(4)invariant operator $\kappa$, which, in matrix form, will convert the labelling scheme $n v\left[\left[f_{v}\right] \times[P P]\right][f]$ into an orthonormal unitary basis for the irreducible representations of $\mathrm{SO}(8) \supset \mathrm{U}(4)$ :

$$
\gamma\left(A^{\dagger}\right)=\kappa^{-1} \Gamma\left(A^{\dagger}\right) \kappa, \quad \gamma(A)=\kappa^{-1} \Gamma(A) \kappa .
$$

The unitarity requirement $\gamma\left(A^{\dagger}\right)=(\gamma(A))^{\dagger}$ and the hermitean adjoint character of $\nabla$ and $z$ leads to

$$
\Gamma\left(A^{\dagger}\right)=\kappa^{2} z \kappa^{-2} .
$$

Again, following ref. ${ }^{1}$ ), a U(4)-invariant operator $\Lambda$ is sought with the property

$$
[\Lambda, z]=\Gamma\left(A^{\dagger}\right)=\kappa^{2} z \kappa^{-2},
$$

where the first step of eq. (23) is used to determine $\Lambda$. The $\Lambda$-operator has the simple form

$$
\begin{aligned}
\Lambda= & -\frac{1}{2} C_{\text {full }}+\frac{1}{4} C_{\text {collective }} \\
& -\frac{1}{4}\left(z_{\sigma} \cdot \nabla_{\sigma}+z_{\tau} \cdot \nabla_{\tau}\right)\left(z_{\sigma} \cdot \nabla_{\sigma}+z_{\tau} \cdot \nabla_{\tau}+2 v-4 \Omega-6\right),
\end{aligned}
$$

where the SU(4) Casimir operators $C_{\text {full }}$ and $C_{\text {collective }}$ are given by eq. (12). In the $\mathrm{SU}(4)$-coupled basis $\left[\left[f_{v}\right] \times[P P]\right]\left[f_{\text {full }}\right]$, with equivalent $\mathrm{SO}(6)$ quantum numbers $\left[\left(p p^{\prime} p^{\prime \prime}\right) \times(P 00)\right]\left(P_{f} P_{f}^{\prime} P_{f}^{\prime \prime}\right)$, the $\Lambda$-operator has the simple eigenvalue

$$
\begin{aligned}
\Lambda_{\text {eigen }}= & -\frac{1}{2}\left(P_{f}\left(P_{f}+4\right)+P_{f}^{\prime}\left(P_{f}^{\prime}+2\right)+P_{f}^{\prime \prime 2}\right) \\
& +\frac{1}{4} P(P+4)-\frac{1}{8}(n-v)\left[\frac{1}{2}(n-v)+2 v-4 \Omega-6\right] .
\end{aligned}
$$

With these eigenvalues, eq. (23) in the form

$$
(\Lambda z-z \Lambda) \kappa^{2}=\kappa^{2} z
$$

leads to recursion relations for the matrix elements of the operator $\kappa^{2}$. Since $\kappa^{2}$ is a 
$\mathrm{U}(4)$-invariant operator, within $\mathrm{SO}(8)$, its matrix elements are diagonal in $v\left[f_{v}\right]$ and $n\left[f_{\text {full }}\right]$, and independent of SU(4) subgroup labels $\mu \equiv \beta S M_{S} T M_{T}$ :

$$
\begin{aligned}
& \left\langle n^{\prime}, v\left[\left[f_{v}\right] \times[P P]\right]\left[f_{\text {full }}^{\prime}\right] \mu^{\prime}\left|\kappa^{2}\right| n, v\left[\left[f_{v}\right] \times[\bar{P} \bar{P}]\right]\left[f_{\text {full }}\right] \mu\right\rangle \\
& =\boldsymbol{\delta}_{n^{\prime} n^{\prime}} \boldsymbol{\delta}_{\left[f_{\text {tull }}^{\prime}\right]\left[f_{\text {full }}\right]} \boldsymbol{\delta}_{\mu^{\prime}, \mu}\left(\kappa^{2}\left(v\left[f_{v}\right] ; n\left[f_{\text {full }}\right]\right)\right)_{P \bar{P}} .
\end{aligned}
$$

The dimension of the $\kappa^{2}$ matrix is thus given by the multiplicity of possible "collective" labcls $P$, i.e. the number of possible $[P P]$ for fixed $v\left[f_{v}\right]$ and a state of definite $n\left[f_{\text {full }}\right]$. There are a large number of interesting cases in the orbital seniority scheme in which the label $P$ is uniquely determined by the quantum numbers $v\left[f_{i}\right]$, $n$ and $\left[f_{\text {full }}\right]$, for which $\kappa^{2}$ is 1-dimensional, e.g. all states of the $\operatorname{SO}(8)$ irreducible representations with $v=0, v=1, v=2$ and $\left[f_{v}\right]=[2], v=3 ;\left[f_{v}\right]=\left[1^{3}\right], v=4$; $\left[f_{v}\right]=\left[1^{4}\right], v=5 ;\left[f_{v}\right]=\left[21^{3}\right], \ldots$, are simple (with 1-dimensional $\kappa^{2}$ matrices). In some $\mathrm{SO}(8)$ irreps certain types of $\mathrm{U}(4)$ irreps lead to simple states only, e.g. in the $\operatorname{SO}(8)$ irrep with $v=2$ and $\left[f_{n}\right]=\left[1^{2}\right]$ states with $\left[f_{\text {full }}\right]=[P+1, P, 1]$ are all simple. For the 1-dimensional case the square-root-taking and inversion of $\kappa^{2}$ required for eq. (21) is trivial. Taking matrix elements of the operator relation, eq. (26), between simple (1-dimensional) states with $n,\left[\int_{\mathrm{R}}\right]$ on the right and $n+2,\left[f_{\mathrm{L}}\right]$ on the left, leads to

$$
\frac{\kappa^{2}\left(v\left[f_{v}\right] ; n+2\left[f_{\mathrm{L}}\right]\right)}{\kappa^{2}\left(v\left[f_{v}\right] ; n\left[f_{\mathrm{R}}\right]\right)}=\Lambda_{\text {eigen }}(\mathrm{L})-\Lambda_{\text {eigen }}(\mathrm{R}),
$$

where the labels $P, \bar{P}$ are ommitted because it is assumed that these are uniquely specified by $n$ and $\left[f_{\mathrm{R}}\right]$ and similarly by $n+2$ and $\left[f_{\mathrm{L}}\right]$. For such states the matrix elements of the unitary realization of the $\mathrm{SO}(8)$ algebra are simply related to the bosonic matrix elements of eq. (20), through

$$
\gamma\left(\boldsymbol{A}^{\dagger}\right)=\kappa z \kappa^{-1}
$$

(see eqs. (21) and (22)). Since the carrier space of bosonic states in the functional representation is mapped isomorphically into the fermion states, eq. (29) also leads directly to the SU(4) reduced matrix elements of $\boldsymbol{A}^{\dagger}\left[\mathrm{cf}\right.$. refs. $\left.{ }^{1.10}\right)$ ]. For the case when both the states on the left and right of the matrix element are simple (with 1-dimensional $\kappa^{2}$ ), eqs. (29) and (28) lead to the general analytic expression for the SU(4) reduced matrix element of $\boldsymbol{A}^{\dagger}$ :

$$
\begin{aligned}
\langle n, v[ & {\left.\left.\left[f_{v}\right] \times[P P]\right]\left[f_{\mathrm{L}}\right]\left\|A^{\dagger}\right\| n-2, v\left[\left[f_{v}\right] \times[\bar{P} \bar{P}]\right]\left[f_{\mathrm{R}}\right]\right\rangle } \\
= & {\left[\Lambda_{\text {eigen }}(\mathrm{L})-\Lambda_{\text {eigen }}(\mathrm{R})\right]^{1 / 2} } \\
& \times\left\langle n, v\left[\left[f_{v}\right] \times[P P]\right]\left[f_{\mathrm{L}}\right]\|z\| n-2, v\left[\left[f_{v}\right] \times[\bar{P} \bar{P}]\right]\left[f_{\mathrm{R}}\right]\right\rangle \\
= & {\left[\frac{1}{2}\left\{P_{\mathrm{R}}\left(P_{\mathrm{R}}+4\right)+P_{\mathrm{R}}^{\prime}\left(P_{\mathrm{R}}^{\prime}+2\right)+P_{\mathrm{R}}^{\prime \prime 2}-P_{\mathrm{L}}\left(P_{\mathrm{L}}+4\right)-P_{\mathrm{L}}^{\prime}\left(P_{\mathrm{L}}^{\prime}+2\right)-P_{\mathrm{L}}^{\prime 2}\right\}\right.} \\
& \left.+\frac{1}{4} P(P+4)-\frac{1}{4} \bar{P}(\bar{P}+4)+\Omega-\frac{1}{4} v-\frac{1}{4} n+\frac{7}{4}\right]^{1 / 2} \\
& \times U\left(\left[f_{v}\right][\bar{P} \bar{P}]\left[f_{\mathrm{L}}\right][11] ;\left[f_{\mathrm{R}}\right][P P]\right)\langle n, P\|z\| n-2, \bar{P}\rangle .
\end{aligned}
$$


TABLE 1

SU(4) reduced matrix elements

$\left\langle n, v\left[\left[f_{v^{\prime}}\right] \times[P P]\right]\left[f_{\mathrm{L}}\right]\left\|\boldsymbol{A}^{\dagger}\right\| n-2, v^{n}\left[\left[f_{v}\right] \times[\bar{P} \bar{P}]\right]\left[f_{\mathrm{R}}\right]\right\rangle$

Case $1:\left(p p^{\prime} p^{\prime \prime}\right)=(000) ; v=0, v=4\left[f_{v}\right]=\left[1^{4}\right] \ldots$

\begin{tabular}{cccc}
\hline$\left[f_{\mathrm{L}}\right]$ & {$\left[f_{\mathrm{R}}\right]$} & $\bar{P}$ & $\left\langle\left\|\boldsymbol{A}^{\dagger}\right\|\right\rangle$ \\
\hline$[P P]$ & {$[P-1, P-1]$} & $P-1$ & $\frac{1}{4}\left[\frac{(4 \Omega-2 P+4-n-v)\left(n+2 P+8-v^{\prime}\right) P}{(P+2)}\right]^{1 / 2}$ \\
{$[P P]$} & {$[P+1, P+1]$} & $P+1$ & $-\frac{1}{4}\left[\frac{(4 \Omega+2 P+12-n-v)(n-2 P-v)(P+4)}{(P+2)}\right]^{1 / 2}$ \\
\hline
\end{tabular}

Case 2: $\left(p p^{\prime} p^{\prime \prime}\right)=\left(\frac{1}{2} \frac{1}{2} \frac{1}{2}\right) ; v=1\left[f_{v}\right]=[1], v=5\left[f_{v}\right]=\left[21^{3}\right], \ldots$

\begin{tabular}{lcccc}
\hline$\left[f_{\mathrm{L}}\right\}$ & {$\left[f_{\mathrm{R}}\right]$} & $\bar{P}$ & $\left\langle\left\|A^{\dagger}\right\|\right\rangle$ \\
\hline$[P+1, P]$ & {$[P, P-1]$} & $P-1$ & $\frac{1}{4}\left[\frac{(4 \Omega-2 P+2-n-v)(n+2 P+8-v) P}{(P+2)}\right]^{1 / 2}$ \\
{$[P+1, P]$} & {$[P+2, P+1]$} & $P+1$ & $-\frac{1}{4}\left[\frac{(4 \Omega+2 P+14-n-v)(n-2 P-v)(P+5)}{(P+3)}\right]^{1 / 2}$ \\
{$[P+1, P]$} & {$[P+1, P+1,1] P+1$} & $-\frac{1}{2}\left[\frac{(4 \Omega-2 P+2-n-v)(n-2 P-v)}{2(P+2)(P+3)}\right]^{1 / 2}$ \\
{$[P, P, 1]$} & {$[P, P-1]$} & $P-1$ & $\frac{1}{2}\left[\frac{(4 \Omega+2 P+10-n-v)(n+2 P+8-v)}{2(P+1)(P+2)}\right]^{1 / 2}$ \\
{$[P, P, 1]$} & {$[P-1, P-1,1] P-1$} & $\frac{1}{4}\left[\frac{(4 \Omega-2 P+6-n-v)(n+2 P+8-v)(P-1)}{(P+1)}\right]^{1 / 2}$ \\
{$[P, P, 1]$} & {$[P+1, P+1,1] P+1$} & $-\frac{1}{4}\left[\frac{(4 \Omega+2 P+10-n-v)(n-2 P-v)(P+4)}{(P+2)}\right]^{1 / 2}$
\end{tabular}

Case 3: $\left(p p^{\prime} p^{\prime \prime}\right)=\left(\frac{1}{2} \frac{1}{2}-\frac{1}{2}\right) ; v=3\left[f_{t}\right]=\left[1^{3}\right], v=7\left[f_{t^{\prime}}\right]=\left[2^{3} 1\right] \ldots$

\begin{tabular}{llll}
\hline$\left[f_{\mathrm{L}}\right]$ & $\left.\mid f_{\mathrm{R}}\right]$ & $\bar{P}$ & $\left\langle\left\|A^{\dagger}\right\|\right\rangle$ \\
\hline$[P, P-1]$ & {$[P-1, P-2]$} & $P-1$ & $\frac{1}{4}\left[\frac{(4 \Omega-2 P+6-n-v)(n+2 P+8-v)(P-1)}{(P+1)}\right]^{1 / 2}$ \\
{$[P, P-1]$} & {$[P P 1]$} & $P-1$ & $\frac{1}{2}\left[\frac{(4 \Omega+2 P+10-n-v)(n+2 P+8-v)}{2(P+1)(P+2)}\right]^{1 / 2}$ \\
{$[P, P-1]$} & {$[P+1, P]$} & $P+1$ & $-\frac{1}{4}\left[\frac{(4 \Omega+2 P+10-n-v)(n-2 P-v)(P+4)}{(P+2)}\right]^{1 / 2}$ \\
{$[P+1, P+1,1]$} & {$[P+1, P]$} & $P+1$ & $-\frac{1}{2}\left[\frac{(4 \Omega-2 P+2-n-v)(n-2 P-v)}{2(P+2)(P+3)}\right]^{1 / 2}$ \\
{$[P+1, P+1,1]$} & {$[P P 1]$} & $P-1$ & $\frac{1}{4}\left[\frac{(4 \Omega-2 P+2-n-v)(n+2 P+8-v) P}{(P+2)}\right]^{1 / 2}$ \\
{$[P+1, P+1,1]$} & {$[P+2, P+2,1]$} & $P+1$ & $-\frac{1}{4}\left[\frac{(4 \Omega+2 P+14-n-v)(n-2 P-v)(P+5)}{(P+3)}\right]^{1 / 2}$
\end{tabular}


TABLE 1 - continued

$$
\begin{aligned}
& \text { Case 4: }\left(p p^{\prime} p^{\prime \prime}\right)=(100) ; v=2,\left[f_{v}\right]=\left[1^{2}\right], v=6\left[f_{u}\right]=\left[2^{2} 1^{2}\right], \ldots \\
& \begin{array}{ccc}
\left.\left[f_{\mathrm{L}}\right]^{\mathrm{a}}\right) & \bar{P} & \left.\left\langle\| f_{\mathrm{R}}\right]^{\mathrm{a}}\right) \\
{[P+1, P, 1][P+2, P+1,1] P+1} & -\frac{1}{4}\left[\frac{(4 \Omega+2 P+12-n-v)(n-2 P-v)(P+3)(P+5)}{(P+2)(P+4)}\right]^{1 / 2} \\
{[P+1, P, 1][P, P-1,1]} & P-1 & \frac{1}{4}\left[\frac{(4 \Omega-2 P+4-n-v)(n+2 P+8-v)(P-1)(P+1)}{P(P+2)}\right]^{1 / 2}
\end{array}
\end{aligned}
$$

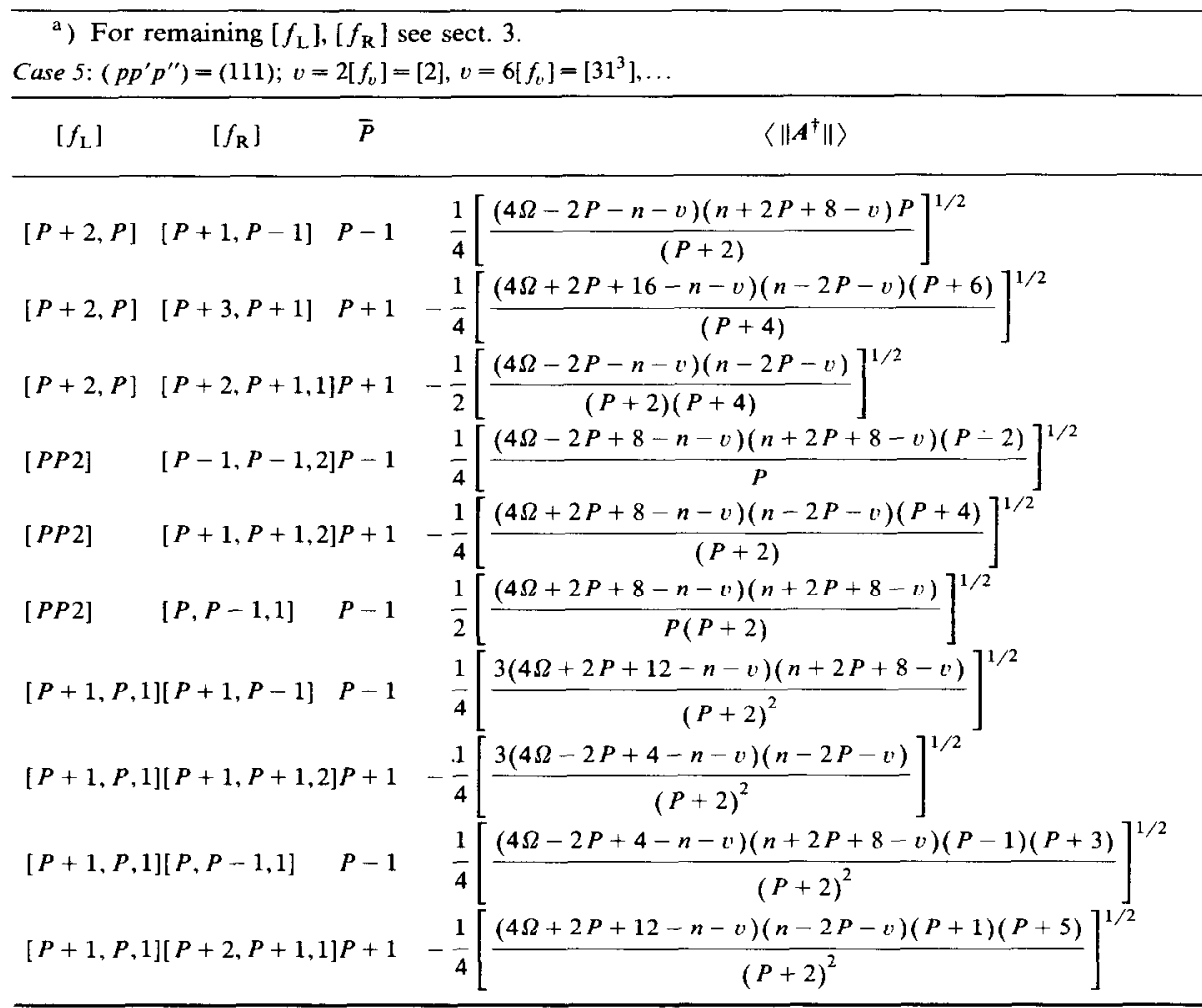

Since the SU(4) Racah coefficients are not widely tabulated, specific results are given in table 1 for some of the cases of greatest applicability for the orbital seniority scheme. [These include as special cases results given for the simple $v=0$ and $v=1$ states in ref. ${ }^{14}$ ); but note that a different phase convention is used in ref. ${ }^{14}$ ). A small printing error from table 4 of ref. ${ }^{14}$ ) has also been corrected.]

\section{The case with multiplicities}

For $S O(8)$ representations and states for which the labels $P$ are not uniquely specified by $n$ and the Wigner-supermultiplet quantum numbers of the $n$-particle 
state the $\kappa^{2}$ operator is best represented in matrix form, as in eq. (27). Its matrix elements can again be evaluated recursively from the matrix form of eq. (26):

$$
\begin{aligned}
\sum_{\bar{P}}[ & \left.\Lambda_{\text {eigen }}\left(n, P,\left[f_{\mathrm{L}}\right]\right)-\Lambda_{\text {eigen }}\left(n-2, \bar{P},\left[f_{\mathrm{R}}\right]\right)\right] \\
\times & \left\langle n\left[\left[f_{v}\right] \times[P P]\right]\left[f_{\mathrm{L}}\right]\|z\| n-2\left[\left[f_{v}\right] \times[\bar{P} \bar{P}]\right]\left[f_{\mathrm{R}}\right]\right\rangle \\
& \times\left(\kappa^{2}\left(v\left[f_{v}\right] ; n-2\left[f_{\mathrm{R}}\right]\right)\right)_{\bar{P} P_{0}} \\
= & \sum_{\hat{P}}\left(\kappa^{2}\left(v\left[f_{v}\right] ; n\left[f_{\mathrm{L}}\right]\right)\right)_{P \hat{P}} \\
& \times\left\langle n\left[\left[f_{v}\right] \times[\hat{P} \hat{P}]\right]\left[f_{L}\right]\|z\| n-2\left[\left[f_{v}\right] \times\left[P_{0} P_{0}\right]\right]\left[f_{\mathrm{R}}\right]\right\rangle .
\end{aligned}
$$

The required square root of the $\kappa^{2}$ operator and its inverse can then be taken if $\kappa^{2}$ is diagonalized via

$$
\sum_{P \bar{P}} O_{i P}^{-1}\left(\kappa^{2}\right)_{P \bar{P}} O_{\bar{P} j}=\lambda_{\imath} \delta_{i j}
$$

where $O_{P i}$ is an orthogonal matrix since all terms of eq. (31) are real. The $\mathrm{SU}(4)$ reduced matrix elements of $\boldsymbol{A}^{\dagger}$ are then given by

$$
\begin{aligned}
\left\langle n, v\left[f_{v}\right] ; i\left[f_{\mathrm{L}}\right]\left\|\boldsymbol{A}^{\dagger}\right\| n-2, v\left[f_{v}\right] ; k\left[f_{\mathrm{R}}\right]\right\rangle \\
=\sum_{P \bar{P}} \kappa_{i P}\left(n\left[f_{\mathrm{L}}\right]\right)\left\langle n\left[\left[f_{v}\right] \times[P P]\right]\left[f_{\mathrm{L}}\right]\|z\| n-2\left[\left[f_{v}\right] \times[\bar{P} \bar{P}]\right]\left[f_{\mathrm{R}}\right]\right\rangle \\
\quad \times \boldsymbol{\kappa}_{\bar{P}{ }^{-1}}{ }^{-1}\left(n-2\left[f_{\mathrm{R}}\right]\right) .
\end{aligned}
$$

The representation $v=2\left[f_{v}\right]=[11]$ is the simplest and yet very useful case where a 2-fold multiplicity occurs. States of the type $[[11] \times[\bar{P} \bar{P}]][P+1, P, 1]$ are still 1-dimensional, with $\bar{P}=P$ only. For these, iteration of eq. (28) gives

$$
\kappa^{2}(v=2[11] ; n[P+1, P, 1])=\frac{\Omega(\Omega-2) !(\Omega+2) !}{(\Omega-P-a-1) !(\Omega+2-a) !}
$$

with $n=2 P+2+4 a$.

States of type $[[11] \times[\bar{P} \bar{P}]][P P]$, however, have a 2-fold multiplicity, with $\bar{P}=P \pm$ 1 . In this case the recursive solution of eq. (31) leads to

$$
\begin{aligned}
& \left(\kappa^{2}(v=2[11] ; n[P P])\right)_{\bar{P} \overline{\bar{P}}}=\frac{(\Omega-2) !(\Omega+1) !}{(P+2)(\Omega-P-k) !(\Omega+2-k) !}
\end{aligned}
$$

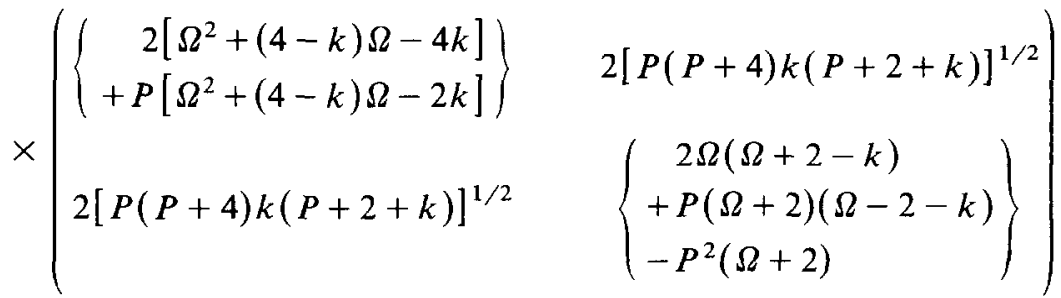


TABLE 2

SU(4) Racah coefficients U([11][PP][PP][11]; [ $\left.\left.f_{12}\right]\left[f_{23}\right]\right)$

\begin{tabular}{|c|c|c|c|c|c|c|c|}
\hline$\left[f_{12}\right] \longrightarrow\left[f_{23}\right]$ & \multicolumn{3}{|c|}{$[P+1, P+1]$} & {$[P-1, P-1]$} & \multicolumn{3}{|c|}{$[P+1, P, 1]$} \\
\hline \multirow{2}{*}[P+1,P+1]{} & \multicolumn{2}{|c|}{2} & & {$[P(P+4)]^{1 / 2}$} & 2 & $P$ & $1 / 2$ \\
\hline & \multicolumn{2}{|c|}{$\overline{(P+1)(P+2)}$} & & $(P+2)$ & $\overline{(P+1)}$ & $\overline{P+2}$ & \\
\hline \multirow{2}{*}[P-1,P-1]{} & \multicolumn{2}{|c|}{$[P(P+4)]^{1 / 2}$} & & 2 & 2 & {$[P+$} & $4]^{1 / 2}$ \\
\hline & \multicolumn{2}{|c|}{$(P+2)$} & & $\overline{(P+2)(P+3)}$ & $(P+3)$ & $\overline{P+}$ & \\
\hline \multirow{2}{*}[P+1,P,1]{} & 2 & $P$ & ]$^{1 / 2}$ & $2[P+4]^{1 / 2}$ & \multicolumn{3}{|c|}{$\left(P^{2}+4 P-1\right)$} \\
\hline & $\overline{(P+1)}$ & $\overline{P+2}$ & & $\overline{(P+3)}[\overline{P+2}$ & \multicolumn{3}{|c|}{$\overline{(P+1)(P+3)}$} \\
\hline
\end{tabular}

with $n=2 P+4 k$. Here $\bar{P}(\overline{\bar{P}})=P+1$ and $P-1$ correspond to the 1 st and 2 nd row (column) of the matrix, respectively. Note that with $k=0, \bar{P}=P-1$ only, and the $\kappa^{2}$ matrix becomes 1-dimensional, with the simple value $\kappa^{2}(v=2[11] ; n=2 P[P P])$ $=(\Omega-2) ! /(\Omega-P-1) !$. It should also be noted that at the boundary of Pauliallowed values, when $n=4 \Omega-2 P$ (or $k=\Omega-P$ ) the $2 \times 2$ matrix has zero determinant, leading to one zero eigenvalue $\lambda$, as required by the fact that these states at the periphery of the Pauli-allowed region must be 1-dimensional. Even so, the expansion of the single allowed state in terms of the U(4)-coupled state vectors will involve states with both of the two possible $\mathrm{U}(4)$ collective labels $\bar{P}=P \pm 1$. (e.g. the coefficient $\langle\bar{P}=P+1 \mid 1\rangle=O_{P+1,1}$, with $|1\rangle \equiv \mid$ allowed state $\rangle$, now has the value $\left.[P(P+4)(\Omega+2) /\{P(P+4)(\Omega+2)+4(\Omega-P)\}]^{1 / 2}\right)$. In the general case eqs. (32)-(35) give the SU(4) reduced matrix elements of the pair-creation operators $A^{\dagger}$. For the evaluation of the reduced matrix elements of $z$, the needed SU(4) Racah coefficients for the special case $\left[f_{v}\right]=[11]$ have the very simple form shown in table 2.

The extended coherent state theory exhibited for the $\mathrm{SO}(8) \supset \mathrm{SU}(4)$ quasispin group in the LST scheme leads to the SU(4) reduced matrix elements of the pair operators in a very simple way, even in cases of challenging seniority quantum numbers. In many of the more challenging cases, however, the $S U(4)$ subgroup labels $S M_{S} T M_{T}$ are insufficient to label the states within the Wigner-supermultiplet scheme. In such cases the SU(4) Wigner coefficients themselves are, in general, not known. The coefficients are needed to complete the construction of states in the orbital seniority scheme. If these coefficients can be evaluated it becomes possible to extend pairing theory in the $L S T$ scheme to include the effects of higher-seniority states. It is the purpose of the next section to exhibit a very general practical method, inspired by the coherent-state construction of this investigation, by which the calculation of $S U(4) \supset[S U(2) \times S U(2)]$ Wigner coefficients can be reduced to tractable form. 


\section{The SU(4) $\supset[S U(2) \times S U(2)]$ Wigner coefficients}

For those irreducible representations where $S M_{S} T M_{T}$ furnish a sufficient set of quantum numbers, the needed $\mathrm{SU}(4) \supset[\mathrm{SU}(2) \times \mathrm{SU}(2)]$ Wigner coefficients are known in general algebraic form ${ }^{17}$ ). Other special cases have also led to analytical expressions involving simple sums ${ }^{18}$ ). The general case is complicated by the problem of "missing" quantum numbers. Although this problem has been solved in principle ${ }^{19.20}$ ), and although projection techniques based on labcls $K_{S}, K_{T}$ have been developed ${ }^{21}$ ) to define a complete basis, it is still a challenging task to calculate the Wigner coefficients in the general case. It is the aim of the present investigation to find a practical effective method to calculate these coefficients, independent of the actual method used to solve the problem of missing quantum numbers.

Since the most general irrep of $\mathrm{SU}(4)$ can be constructed from the SU(4) product $[P P] \times[y] \rightarrow[P+x, P, y-x]$ where $[y]$ is the totally symmetric representation, the most general SU(4) representation can be constructed from products of "collective" states, such as those of eq. (14), and "intrinsic" states belonging to the totally symmetric irrep of $\mathrm{SU}(4)$. It is useful to define the $\mathrm{SU}(2)$-coupled state vectors

$$
\begin{aligned}
\left|[P P],[y] ;\left[S_{P} s\right] S M_{S}\left[T_{P} t\right] T M_{T}\right\rangle= & \sum_{M_{S_{P}} m_{s}} \sum_{M_{T_{P}} m_{t}}\left|[P P] S_{P} M_{S_{P}} T_{P} M_{T_{P}}\right\rangle\left|[y] s m_{s} t m_{t}\right\rangle \\
& \times\left\langle S_{P} M_{S_{P}} s m_{s} \mid S M_{S}\right\rangle\left\langle T_{P} M_{T_{P}} t m_{t} \mid T M_{T}\right\rangle
\end{aligned}
$$

Note that $t=s$ within the totally symmetric representation $[y]$. The SU(4) eigenstates can then be expanded in terms of this basis:

$$
\begin{aligned}
& \left|[f] \beta S M_{S} T M_{T}\right\rangle \\
& \quad=\sum_{S_{P} T_{P}} \sum_{s=t}\left|[P P],[y] ;\left[S_{P} s\right] S M_{S}\left[T_{P} s\right] T M_{T}\right\rangle c_{\left[S_{P} s\right] S,\left[T_{P} s\right] T}^{[P P \| y] f] \beta},
\end{aligned}
$$

where $\beta$ distinguishes states with a multiple occurrence of a particular pair $S, T$. To ensure that the state (37) belongs to a particular SU(4) irreducible representation $[f]$, it is sufficient to diagonalize the operator (cf. eq. (12a)):

$$
C-C_{\text {intr }}-C_{\text {coll }}=(\boldsymbol{\sigma} \tau)_{\alpha \beta}\left(z_{\sigma \alpha} \nabla_{\tau \beta}+z_{\tau \beta} \nabla_{\sigma \alpha}\right)+2\left(\sigma_{\text {intr }} \cdot S_{\text {coll }}\right)+2\left(t_{\text {intr }} \cdot T_{\text {coll }}\right) .
$$

With $[f]=[P+x, P, y-x]$, this must now have the eigenvalue

$$
\left(P+x-\frac{1}{2} y\right)\left(P+x-\frac{1}{2} y+4\right)+\frac{1}{2} y\left(\frac{1}{2} y+2\right)+\left(x-\frac{1}{2} y\right)^{2}-P(P+4)-\frac{3}{4} y(y+4) .
$$

Since the operators $\left(\delta_{\text {intr }} \cdot S_{\text {coll }}\right)$ and $\left(\ell_{\text {intr }} \cdot \boldsymbol{T}_{\text {coll }}\right)$ are diagonal in the basis (36) with 
trivial eigenvalues, it is sufficient to evaluate the matrix elements of $(\sigma \tau)_{\alpha \beta}\left(z_{\sigma \alpha} \nabla_{\tau \beta}+\right.$ $\left.z_{\tau \beta} \nabla_{\sigma \alpha}\right)$ in this basis. Straightforward angular momentum coupling theory gives

$$
\begin{aligned}
& \left\langle[P P],[y] ;\left[S_{P}^{\prime} s^{\prime}\right] S M_{S}\left[T_{P}^{\prime} s^{\prime}\right] T M_{T}\right|(\sigma \tau)_{\alpha \beta}\left(z_{\sigma \alpha} \nabla_{\tau \beta}+z_{\tau \beta} \nabla_{\sigma \alpha}\right) \\
& \times\left|[P P],[y] ;\left[S_{P} s\right] S M_{S}\left[T_{P} s\right] T M_{T}\right\rangle \\
& =\frac{\left(2 s^{\prime}+1\right)}{(2 s+1)} U\left(S S_{P} s^{\prime} 1 ; s S_{P}^{\prime}\right) U\left(T T_{P} s^{\prime} 1 ; s T_{P}^{\prime}\right)
\end{aligned}
$$

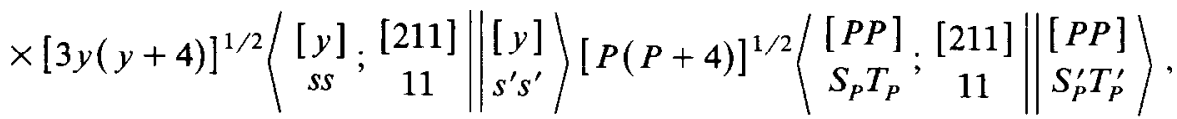

where the double-barred coefficients are $\mathrm{SU}(4) \supset[\mathrm{SU}(2) \times \mathrm{SU}(2)]$ reduced Wigner coefficients. For the representations $[P P]$ and $[y]$ these are known in general algebraic form and can be read from tables $A 4.1$ and $A 4.2$ of ref. ${ }^{17}$ ). In those cases where the additional quantum number $\beta$ is needed the diagonalization of the operator $C-C_{\text {intr }}-C_{\text {coll }}$ leads to a set of $d$ solutions, where $d$ is the multiplicity of the state $(\beta=1, \ldots, d)$. In a practical method of calculation it has been found most effective to choose these $d$ solutions in some arbitrary way and convert the labeled states $\beta$ to eigenstates of a physically interesting operator at the very last stage of the calculation.

The $v=2$ states which are of particular interest for $L S T$ pairing theory include the SU(4) irreps $[P+2, P],[P+1, P, 1]$ and $[P P 2]$, see table 1 . These irreps all include possible 2-fold occurrences for certain $S, T$ values and will be used as the prime example to illustrate the proposed method of calculation. Since the set of possible $S_{P} T_{P}$ values of the basis (37) depends on the parity of $S+T$, the two cases $P-S-T=$ even/odd must be treated separately. For the case $P-S-T=$ odd, 2-fold multiplicities can occur only in the representation $[P+1, P, 1]$. Diagonalization of $C-C_{\text {intr }}-C_{\text {coll }}$ leads to the set of expansion coefficients $c^{[f] \beta}$ shown in table 3. The two independent solutions for $[f]=[P+1, P, 1]$ have been chosen arbitrarily; in this case, by setting one of the $c$ 's equal to zero for the statc labelcd $\beta=1$. For convenience, the states $\beta=1$ and 2 have been made orthogonal to each other; but it is not necessary to orthogonalize the basis at this stage of the calculation. To convert the states labeled $\beta=1$ and 2 to a more meaningful basis, it is now straightforward to find the eigenvectors of the operators $\Omega$ and $\Phi$ proposed by Moshinsky and Nagel ${ }^{19}$ ), or some other set of operators. For the states of table 3 , the operator $\Omega$ is sufficient. It can be expressed in terms of intrinsic and collective operators:

$$
\Omega_{\mathrm{op}}=\sum_{\alpha, \beta}\left\{\left(f s_{\mathrm{intr}}+\boldsymbol{S}_{\mathrm{coll}}\right)_{\alpha}\left(\frac{1}{2}(\boldsymbol{\sigma} \tau)_{\alpha \beta}+z_{\sigma \alpha} \nabla_{\tau \beta}+z_{\tau \beta} \nabla_{\sigma \alpha}\right)\left(f t_{\mathrm{intr}}+\boldsymbol{T}_{\mathrm{coll}}\right)_{\beta}\right\}
$$




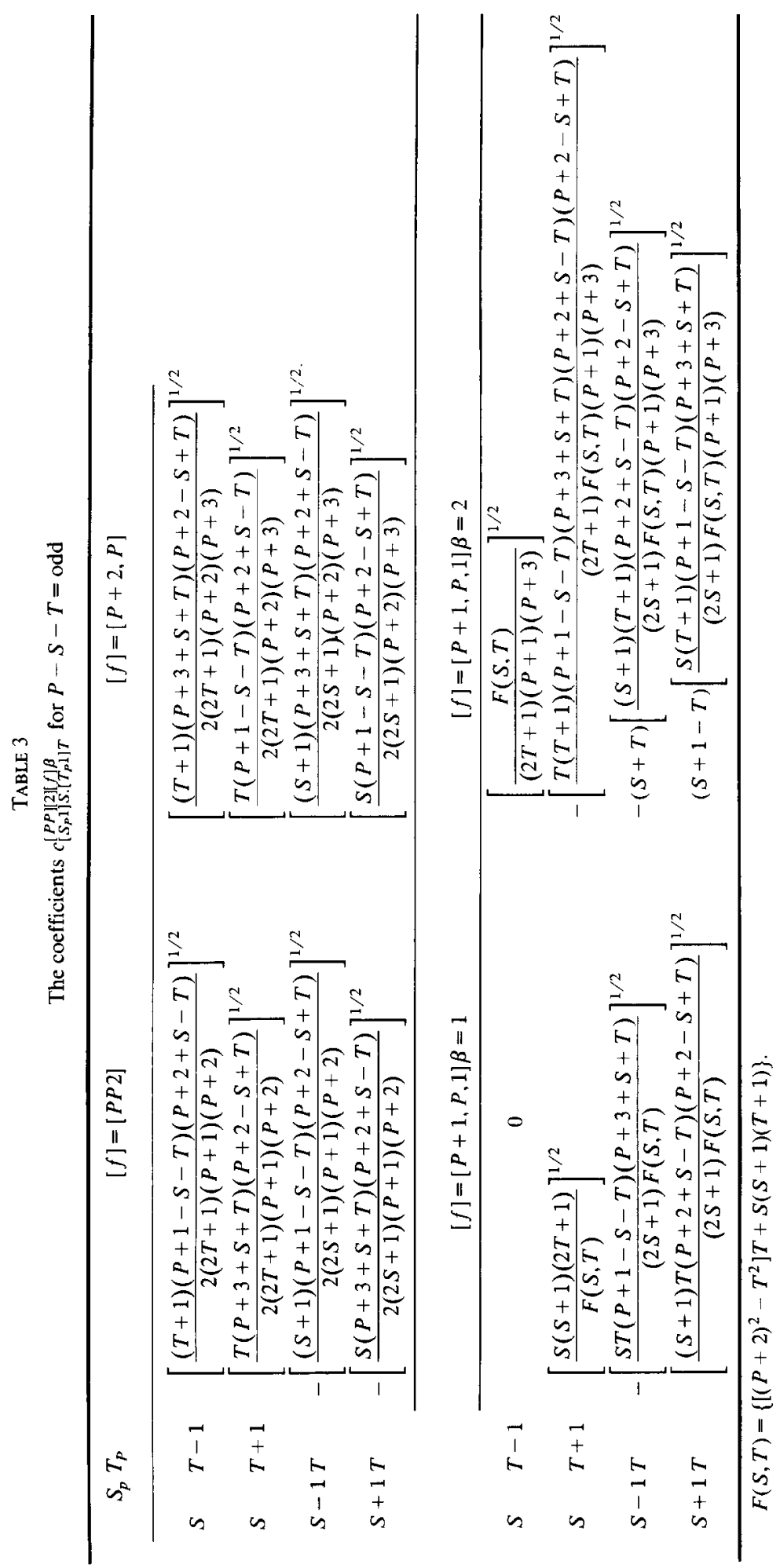


In the basis (36) it has the matrix elements

$$
\begin{aligned}
& \left\langle[P P],[y] ;\left[S_{p}^{\prime} s^{\prime}\right] S M_{S}\left[T_{P}^{\prime} s^{\prime}\right] T M_{T}\left|\Omega_{\mathrm{op}}\right|[P P],[y] ;\left[S_{P} s\right] S M_{S}\left[T_{P} s\right] T M_{T}\right\rangle \\
& =\delta_{S_{P}^{\prime} S_{P}} \delta_{T_{P}^{\prime} T_{P}}\left\{\delta_{s s^{\prime}} \frac{1}{4}(y+2)\left[S_{P}\left(S_{P}+1\right)-S(S+1)+T_{P}\left(T_{P}+1\right)-T(T+1)\right]\right. \\
& +U\left(S S_{P} s^{\prime} 1 ; s S_{P}\right) U\left(T T_{P} s^{\prime} 1 ; s T_{P}\right)\left[S_{P}\left(S_{P}+1\right) T_{P}\left(T_{P}+1\right)\right]^{1 / 2} \\
& \left.\times \frac{\left(2 s^{\prime}+1\right)}{(2 s+1)}\left[\frac{3 y(y+4)}{4}\right]^{1 / 2}\left\langle\begin{array}{c}
{[y] ;} \\
s s
\end{array} ; \begin{array}{c}
{[211]} \\
11
\end{array} \| \begin{array}{l}
{[y]} \\
s^{\prime} s^{\prime}
\end{array}\right)\right\} \\
& +\delta_{s s^{\prime}} \cdot 2 U\left(S S_{P} s 1 ; s S_{P}^{\prime}\right) U\left(T T_{P} s 1 ; s T_{P}^{\prime}\right) s(s+1) \\
& \times[P(P+4)]^{1 / 2}\left(\begin{array}{cc||c|c}
{[P P]} & {[211]} \\
S_{P} T_{P} & 11 & {[P P]} \\
S_{P}^{\prime} T_{P}^{\prime}
\end{array}\right),
\end{aligned}
$$

where the necded doublc-barred coefficients can again be read from tables A4.1 and A4.2 of ref. $\left.{ }^{17}\right)$. For the irreducible representation $[P+1, P, 1]$ and states with $P-S-T=$ odd (see table 3 ) this leads to the simple eigenvalues

$$
\begin{aligned}
\Omega_{\text {eigen }}= & \frac{1}{(P+1)(P+3)} \\
& \times\{T(T+1)+S(S+1) \pm[ \\
& \quad+4(T+1)+S(S+1)]^{2} \\
& \left.+4 S+1) T(T+1)(P+1)(P+3)]^{1 / 2}\right\} .
\end{aligned}
$$

Despite the simple algebraic form of $\Omega_{\text {eigen }}$, the eigenvectors of $\Omega_{\mathrm{op}}$ have a more complicated algebraic structure than the states $\beta=1$ and 2 of table 3 .

For the states with $P-S-T=$ even, all three representations $[P, P+2],[P+$ $1, P, 1]$, and $[P P 2]$ may now have 2 -fold multiplicities. The $c^{[f] \beta}$ for the two solutions $\beta=1$ and $\beta=2$ are now restricted by the relations

$$
\begin{aligned}
& {[S(T+1)]^{1 / 2} c_{00}-[(S+1) T]^{1 / 2} c_{11}} \\
& \quad=c_{-+}\left[\frac{(2 S+1)(2 T+1)}{(P+3-S+T)(P+1+S-T)}\right]^{1 / 2}\left\{\begin{array}{c}
-(P+3-S+T) \\
(S-T) \\
(P+1+S-T)
\end{array}\right\}, \\
& {[(S+1) T]^{1 / 2} c_{00}-[S(T+1)]^{1 / 2} c_{11}} \\
& \quad=c_{+}\left[\frac{(2 S+1)(2 T+1)}{(P+3+S-T)(P+1-S+T)}\right]^{1 / 2}\left\{\begin{array}{c}
-(P+3+S-T) \\
-(S-T) \\
(P+1-S+T)
\end{array}\right\},
\end{aligned}
$$




$$
\begin{aligned}
& -[S T]^{1 / 2} c_{00}-[(S+1)(T+1)]^{1 / 2} c_{11} \\
& =c_{--}\left[\frac{(2 S+1)(2 T+1)}{(P+2-S-T)(P+2+S+T)}\right]^{1 / 2}\left\{\begin{array}{c}
-(P+2-S-T) \\
(S+T+1) \\
(P+2+S+T)
\end{array}\right\}, \\
& -[(S+1)(T+1)]^{1 / 2} c_{00}-[S T]^{1 / 2} c_{11} \\
& =c_{++}\left[\frac{(2 S+1)(2 T+1)}{(P-S-T)(P+4+S+T)}\right]^{1 / 2}\left\{\begin{array}{c}
-(P+4+S+T) \\
-(S+T+1) \\
(P-S-T)
\end{array}\right\},
\end{aligned}
$$

with the shorthand notation for the $c_{\left[S_{P} s\right] S,\left[T_{p} s\right] T}^{[P[2] f]}$ :

$$
\begin{array}{rlrl}
c_{00} & \equiv c_{[S 0] S,[T 0] T}^{P P \|[2] f]}, & c_{11} \equiv c_{[S 1] S,[T 1] T}^{[P P[[2][f]}, \\
c_{-+} & \equiv c_{[S-1,1] S,[T+1,1] T}^{P P][2][f]}, \text { etc. }
\end{array}
$$

In eqs. (43) lines 1,2 , and 3 correspond to the irreps $[P+2, P],[P+1, P, 1]$, and $[P P 2]$ in that order. Although it is possible to construct two independent solutions to eqs. (43), e.g. by choosing $c_{00}=0$ to construct the state to be labeled $\beta=1$, the algebraic structure of the resulting states is rather complicated. In practice, however, it is straightforward to find solutions to eqs. (43) which are also eigenfunctions of $\Omega_{\text {op }}$ for any specific pair of $S, T$ values, e.g. with $P=2, S, T=1,1$ and $[f]=[321]$, the two states with

$$
\begin{aligned}
& c_{[21] 1,[01] 1}^{[22[1] 132] 1}=\sqrt{\frac{1}{2}}, \quad c_{[01] 1,[21] 1}^{[22][2][32] 1}=-\sqrt{\frac{1}{2}},
\end{aligned}
$$

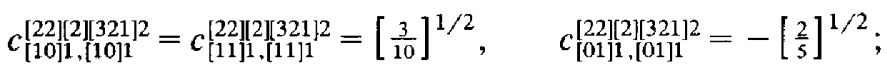

are eigenstates of $\Omega_{\mathrm{op}}$ with eigenvalues $2, \frac{1}{10}$ for $\beta=1$ and 2 .

Once the $c$ 's have been determined to define the state vectors $\left.\| f] \beta S M_{S} T M_{T}\right\rangle$ in the SU(2)-coupled basis of eq. (37), it is straightforward to evaluate the needed $\mathrm{SU}(4) \supset \mathrm{SU}(2) \times \mathrm{SU}(2)$ Wigner coefficients. For example, all the $\mathrm{SU}(4)$ Wigner coefficients needed for cases 5 of table 1 can be determined from the matrix elements of a unit tensor operator of collective type of $\operatorname{rank}[11]$ with $S T=10$ or 01 . With $S T=10$ this leads to

$$
\begin{aligned}
& \left\langle\begin{array}{ll||l}
{[f]} & {[11]} & {\left[f^{\prime}\right]} \\
\beta S T ; & 10 & \beta^{\prime} S^{\prime} T
\end{array}\right\rangle
\end{aligned}
$$

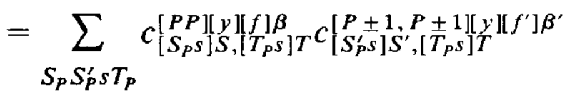

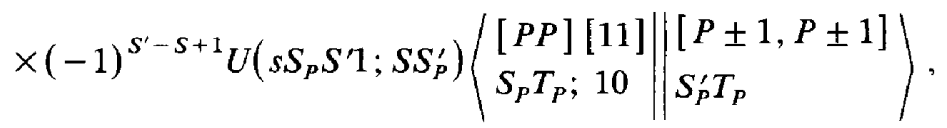

with an analagous expression if [11]10 is replaced by [11]01. In eq. (45) the 
double-barred SU(4) Wigner coefficients of the right-hand side are known in general algebraic form and can be read from table A2.1 of ref. ${ }^{17}$ ). It may also be useful to use a unit tensor operator of intrinsic type and rank $[1] \frac{1}{2} \frac{1}{2}$, which leads to

$$
\begin{aligned}
& \left.\left\langle\begin{array}{ll||c}
{[f]} & {[11]} \\
\beta S T ; & \frac{1}{2} \frac{1}{2}
\end{array}\right| \begin{array}{c}
{\left[f^{\prime}\right]} \\
\beta^{\prime} S^{\prime} T^{\prime}
\end{array}\right)
\end{aligned}
$$

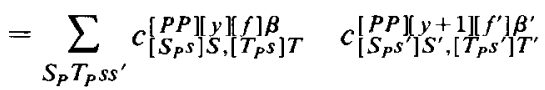

$$
\begin{aligned}
& \left.\times U\left(S_{P} S S^{\prime} \frac{1}{2} ; S s^{\prime}\right) U\left(T_{P} s T^{\prime} \frac{1}{2} ; T s^{\prime}\right)\left\langle\begin{array}{l}
{[y][1]} \\
s s ; \frac{1}{2} \frac{1}{2}
\end{array}\right| \begin{array}{l}
{[y+1]} \\
s^{\prime} s^{\prime}
\end{array}\right),
\end{aligned}
$$

where the double-barred coefficient of the right-hand side of eq. (46) can be read from table A1.4 of ref. ${ }^{17}$ ). SU(4) Wigner coefficients with more complicated couplings can also be evaluated from those of eq. (46) by the buildup method employed in ref. ${ }^{17}$ ).

In conclusion, it can be stated that the generalized coherent-state techniques, used recently to great advantage for the very explicit construction of an orthonormal unitary basis for the noncompact groups $\operatorname{Sp}(2 d, \mathrm{R}) \supset \mathrm{U}(d)$, can be used with equal facility for a complete construction of the matrix elements of the LST quasispin group. Since the needed $\mathrm{SU}(4) \supset[\mathrm{SU}(2) \times \mathrm{SU}(2)]$ subgroup algebra is worked out in sufficient detail, this method leads to a more explicit construction of many-nucleon states in the orbital seniority scheme and a classification in terms of nucleon-number and Wigner-supermultiplet symmetry. Although extensions to more complicated noninvariance groups are possible and have many interesting nuclear physics applications ${ }^{11}$ ), very explicit constructions may be possible only in those cases where the Wigner-Racah calculus for the intrinsic subgroup can be worked out in sufficient detail.

\section{References}

1) D.J. Rowe, J. Math. Phys. 25 (1984) 2662;

D.J. Rowe, G. Rosensteel and R. Carr, J. of Phys. A17 (1984) L399:

G. Rosensteel and D.J. Rowe, Int. J. Theor. Phys. 16 (1977) 63

2) O. Castaños, E. Chacón and M. Moshinsky, J. Math. Phys. 25 (1984) 1211;

O. Castaños, E. Chacõn, M. Moshinsky and C. Quesne, J. Math. Phys., to be published;

M. Moshinsky, J. of Phys. A18 (1985) L1

3) J. Deenen and C. Quesne, J. Math. Phys. 25 (1984) 2354; Université de Bruxelles report PTM 84-07; J. of Phys. A17 (1984) L405

4) P. Kramer, Ann. of Phys. 141 (1982) 259

5) G. Rosensteel and D.J. Rowe, Phys. Rev. Lett. 38 (1977) 10; Ann. of Phys. 126 (1980) 343

6) F. Arickx, Nucl. Phys. A268 (1976) 347;

F. Arickx, J. Broeckhove and E. Deumens, Nucl. Phys. A318 (1979) 269

7) P. Park, J. Carvalho, M. Vassanji, D.J. Rowe and G. Rosensteel, Nucl. Phys A414 (1984) 93;

J.P. Draayer, K.J. Weeks and G. Rosensteel, Nucl. Phys. A413 (1984) 215

8) J. Dobaczewski, Nucl. Phys. A369 (1981) 213 (I), 237 (II); A380 (1982) 1 (III) 
9) M. Moshinsky and C. Quesne, J. Math. Phys. 11 (1970) 1631; 12 (1971) 1772;

B.R. Judd, in Group theory and applications, ed. E.M. Loebl (Academic, New York, 1968)

10) K.T. Hecht and J.P. Elliott, Nucl. Phys. A438 (1985) 29

11) K.T. Hecht, Proc. VIII Symp. on nuclear physics, Oaxtepec, Mexico (1985)

12) J. Ginocchio, Ann. of Phys. 126 (1980) 234

13) B.H. Flowers and S. Szpikowski, Proc. Phys. Soc. 84 (1964) 193, 673

14) Sing Chin Pang, Nucl. Phys. A128 (1969) 497

15) P. Kramer, G. John and D. Schenzle, Group theory and the interaction of composite nucleon systems (Vieweg, Braunschweig 1981) ch. 4

16) K.T. Hecht and Y. Suzuki, J. Math. Phys. 24 (1983) 785

17) K.T. Hecht and Sing Chin Pang, J. Math. Phys. 10 (1969) 1571

18) S. Alisauskas, J. of Phys. A17 (1984) 2899; Liet. Fiz. Rink. 20 (1980) Nr. 2, 3; 23 (1983) Nr. 3, 3

19) M. Moshinsky and J.G. Nagel, Phys. Lett. 5 (1963) 173

20) M. Brunet and M. Resnikoff, J. Math. Phys. 11 (1970) 1471, 1474

21) J.P. Draayer, J. Math. Phys. 11 (1970) 3225 